\title{
A gloves-associated outbreak of imipenem-resistant Acinetobacter baumannii in an intensive care unit in Guangdong, China
}

\author{
Dan $\mathrm{Ye}^{1}$, Jinglan Shan ${ }^{1}$, Yongbo Huang ${ }^{2,3}$, Jianchun $\mathrm{Li}^{2,3}$, Changan $\mathrm{Li}^{1}$, Xiaoqing $\mathrm{Li}^{2,3}$, Weiqun $\mathrm{He}^{2,3}$, Yimin $\mathrm{Li}^{2,3^{*}}$ \\ and Pu $\mathrm{MaO}^{2^{*}}$
}

\begin{abstract}
Background: Imipenem-resistant Acinetobacter baumannii (IRAB) is an important cause of hospital-acquired infection. We aimed to describe an outbreak of IRAB infection and to investigate its possible source in an intensive care unit.

Methods: An environmental investigation was undertaken. Antimicrobial susceptibility testing was performed by microdilution. These isolates were genotyped by use of repetitive extragenic palindromic polymerase chain reaction (rep-PCR; DiversiLab). The study included 11 patients infected with IRAB and 14 control patients free of IRAB. Case and control patients were compared for possible predisposing factors. A multifaceted intervention was implemented to control the outbreak.
\end{abstract}

Results: Thirty-nine IRABs were isolated from patients and the environmental surveillance culture in August, November, and December 2011. All isolates were resistant to imipenem. The IRAB strains belonged to seven clones (A-G) by the use of rep-PCR. There were four epidemic clones $(D-G)$ in the outbreak, and Clone $D$ was predominant. For the case-control study, patients with chronic obstructive pulmonary disease were susceptible to infection with IRAB. The hospital mortality of the case group was significantly higher than that of the control group.

Conclusions: The outbreak strains were transmitted among infected patients and equipment by inappropriate use of gloves. A combination of aggressive infection control measures is essential for preventing recurrent nosocomial outbreaks of IRAB.

Keywords: Outbreak, Acinetobacter baumannii, ICU, Gloves

\section{Background}

Acinetobacter baumannii, a non-fermenting Gram-negative bacterium, is recognized as an important opportunistic pathogen, and is particularly associated with mortality in intensive care units (ICUs) [1]. As a result of the simplicity of its growth requirements and its remarkable capacity for extended survival on environmental surfaces, A. baumannii is ubiquitous in the environment [2]. Thus, environmental contamination is an important source of cross-infection $[3,4]$. The carbapenem group of antimicrobial agents is commonly used for treating nosocomial infections

\footnotetext{
* Correspondence: dryiminli@vip.163.com; maopu1981@126.com ${ }^{2}$ State Key Laboratory of Respiratory Disease, The First Affiliated Hospital of Guangzhou medical university, Guangzhou, Guangdong 510120, China ${ }^{3}$ Intensive Care Unit, The First Affiliated Hospital of Guangzhou medical university, Guangzhou, Guangdong 510120, China

Full list of author information is available at the end of the article
}

caused by A. baumannii [5]. However, carbapenem resistance has been increasingly identified in the past decade [6,7], and imipenem-resistant A. baumannii (IRAB) has also been increasingly reported as a cause of nosocomial outbreaks [8-12].

On August 9, 2011, an outbreak of nosocomial infection with IRAB was noted in our medical ICU. In this study, we isolated IRAB from clinical specimens and the hospital environment, using the DiversiLab repetitive extragenic palindromic sequence-based PCR (rep-PCR) to assess the genetic relationship of these resistant isolates.

\section{Methods}

The study was approved by the institutional Research Ethical Board of the First Affiliated Hospital

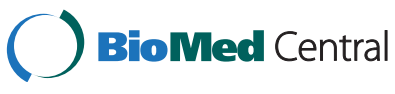

(c) 2015 Dan et al.; licensee BioMed Central. This is an Open Access article distributed under the terms of the Creative Commons Attribution License (http://creativecommons.org/licenses/by/4.0), which permits unrestricted use, distribution, and reproduction in any medium, provided the original work is properly credited. The Creative Commons Public Domain Dedication waiver (http://creativecommons.org/publicdomain/zero/1.0/) applies to the data made available in this article, unless otherwise stated. 
of Guangzhou Medical University (2010082) for implementing outbreak control interventions and collecting clinical data retrospectively.

\section{Hospital settings}

The First Affiliated Hospital of Guangzhou Medical University is a 2,000-bed, teaching hospital, and a tertiary referral center for major respiratory diseases in Southern China. In 2011, the annual number of admissions to the First Affiliated Hospital of Guangzhou Medical University was 45,020 and there were 560 admissions to the ICU. The outbreak occurred in a 27-bed medical ICU, where the nurse-to-patient ratio was 1-1.5:1 during daytime and 1:3 at night. A map of the ICU is shown in Figure 1.

\section{Epidemiological investigation}

The outbreak period was defined as August 1-23. Nosocomial infection was defined according to the Centers for Disease Control and Prevention (CDC) [13]. For the case-control study, the cases were defined as those who had stayed in the ICU for at least $48 \mathrm{~h}$ with IRAB infection. The controls were patients without identification of IRAB after screening twice during the study period. Case and control patients were screened twice every week.

Demographic (age and sex) and clinical data (duration of ICU and hospital stay, sites of infection or colonization, underlying diseases, diagnosis, antibiotic classes used, mechanical ventilation, blood transfusion, hemodialysis, SOFA score, APACHE II urinary catheter, nasogastric

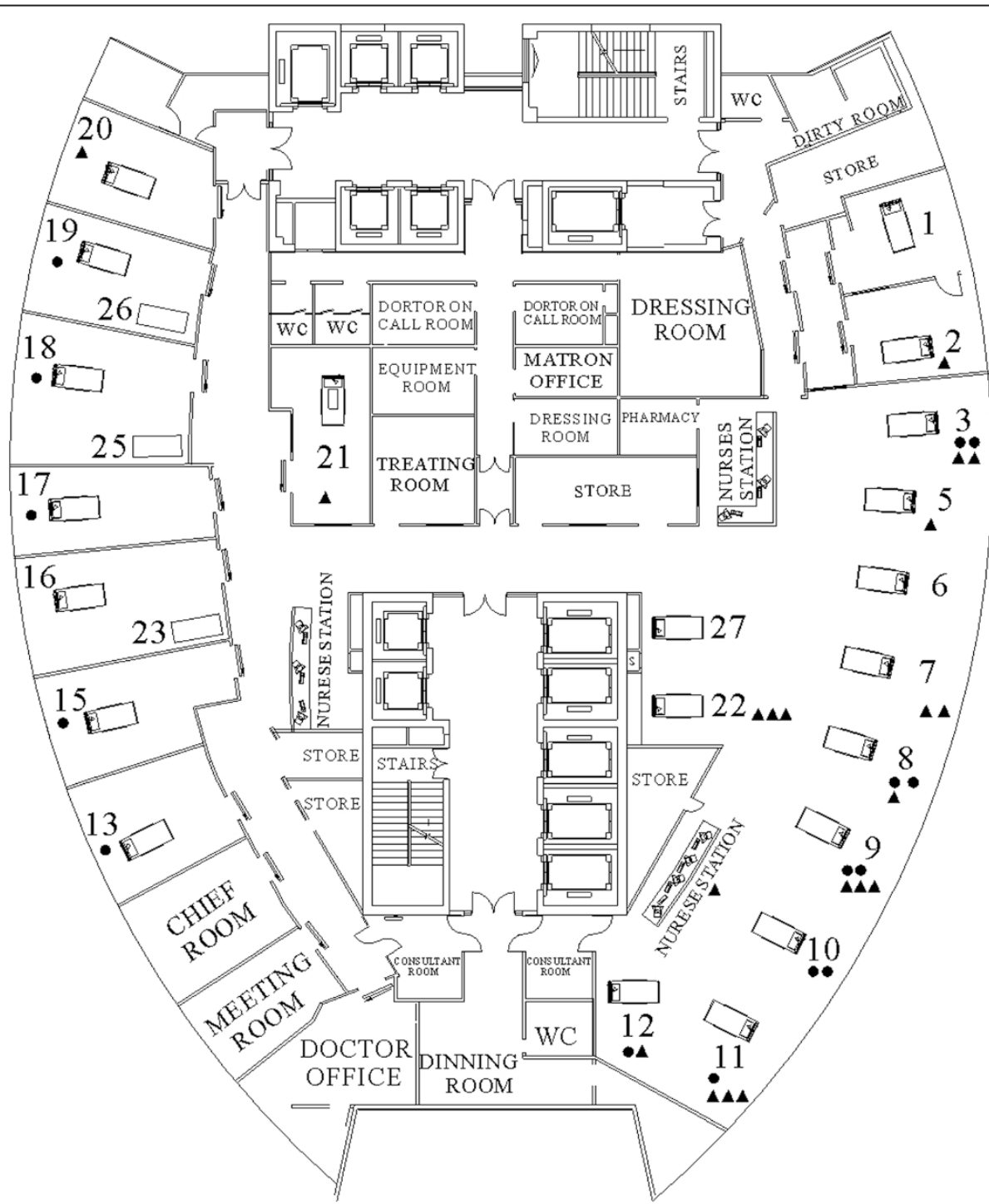

Figure 1 Schematic map of ICU showing the locations of samples that were positive for IRAB. (•) Patients with isolates positive for IRAB during environmental surveys in August, November, and December, 2011. (A) Environmental samples positive for IRAB during environmental surveys in August, November, and December, 2011. 
tube, central venous catheter, peripheral venous catheter, arterial catheter, and clinical outcome) were extracted from the patient medical records.

\section{Microbiological methods}

Research assistants swabbed potential fomites in the patient environment during outbreaks. Cotton-tipped swabs were moistened with sterile saline and surfaces vigorously scrubbed. Potential fomites included ventilator control panel and sensor, bed rails, supply cabinets, computer keyboards and mice, curtains, air vents, door handles, supply carts, and mattresses.

Transtracheal aspiration specimens were inoculated onto blood agar and MacConkey agar and incubated at $37^{\circ} \mathrm{C}$ for $48 \mathrm{~h}$ in an air incubator. Environmental swabs were incubated overnight in fastidious broth and then subcultured onto blood agar and MacConkey agar. Identification was conducted using the VITEK2 Compact 30 System (bioMerieux). The antimicrobial susceptibility was determined by microdilution in accordance with the Clinical and Laboratory Standards Institute guidelines.

\section{Molecular typing}

Epidemiological typing of isolates was performed by rep-PCR fingerprinting (DiversiLab System) as previously described. Data were sent via an individual secured website (https://gzmedical.diversilab.com/) and analyzed with DiversiLab version 3.5 , using the Pearson correlation coefficient and the unweighted pair group method. The isolates clustered $\geq 95 \%$ was considered related.

\section{Infection control interventions}

Standard infection control measures were reinforced during the early outbreak (August 1-11). These included re-emphasizing hand hygiene; using alcohol foam between patient contacts following five moments of hand hygiene practices as recommended by the World Health Organization; and the practice of sterile techniques for all invasive procedures.

The crisis control staff decided to implement the enhanced control interventions, after five new cases were diagnosed during the early outbreak. Enhanced control interventions were implemented. (1) A cohort strategy was implemented. Colonized/infected patients were separated into different areas. The newly admitted patients were also separated and treated by a different team of healthcare workers (HCWs). (2) Inappropriate wearing of gloves in the ICU was strictly prohibited, and violators were financially penalized. One month merit pay should be kept out to pay for the reeducation. (3) The ICU was divided into two zones for daily cleaning, with separation of the clinical area and the HCW living area. The cleaning and decontamination criteria were formulated. The performance of regular environmental cleaning procedures in every shift was evaluated. (4) Unnecessary transfer of patients from other units or surrounding hospitals was stopped. Patients no longer requiring intensive care were immediately transferred to another unit with contact precautions. (5) Contact precautions were practiced for all patients upon admission. Continuation of contact precautions during ICU stay depended on the detection of IRAB in the first culture result. (6) Education crisis control staff reported progress of the outbreak and emphasized infection control lapses during the doctors' and nurses' shifts. The cleaning staffs were retrained.

Written informed consent for participation in the study was obtained from all participants.

\section{Statistical analysis}

Continuous variables were described as mean and standard deviation, or median and interquartile range if they showed a skewed distribution. Categorical variables were described with absolute percentages. Continuous variables were analyzed by the Student's $t$-test or the Mann-Whitney $U$ test for non-parametric distributions. The categorical variables were compared by using the Pearson $\chi^{2}$ test.

\section{Results}

\section{Outbreak description}

In mid-August 2011, the Infection Control Department was notified that there was an outbreak of IRAB infection. During the outbreak period, 11 patients had IRABpositive cultures isolated from the lower respiratory tract. The mean age of affected patients was 65 years (range: $39-85$ years). The antibiotic susceptibility patterns of the clinical isolates were identical, and the isolates were resistant to the majority of antibiotics, including carbapenems. An outbreak control team was established including ICU doctors and a head nurse, bacteriologists, infection control officer, and nurses. At the beginning of the outbreak, covert observation found that some doctors and nurses were wearing gloves all the time. The timeline of the outbreak investigation is illustrated in Figure 2.

\section{Isolation of IRAB}

The distribution of patients with IRAB acquisition in the ICU and imported to the ICU during 2011 is shown in Figure 3 . The number of patients that acquired IRAB in the ICU increased with the number of imported patients with IRAB. The majority of patients who were admitted to the ICU had pulmonary disease [severe pneumonia $16 / 25$, and chronic obstructive pulmonary disease (COPD) 12/25], and all were treated with mechanical ventilation, therefore, we focused our analysis on bacteria 


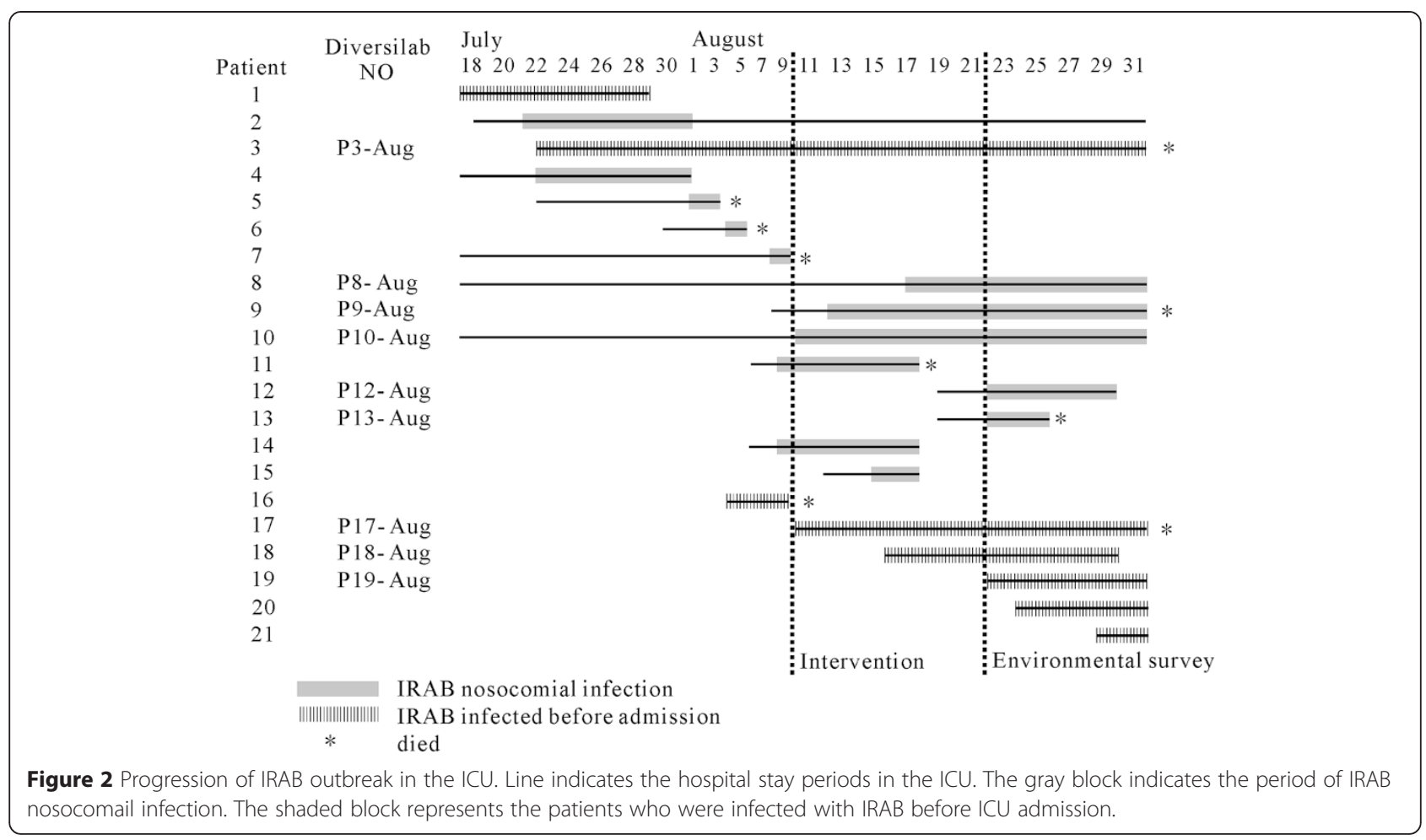

isolated from transtracheal aspirates. These IRAB strains were also resistant to commonly used antibiotics, except polymyxin B and colistin. All of these strains were multidrug resistant.

\section{Case-control study}

The demographic risk factors for nosocomial acquisition of IRAB and outcome were compared between the case and control groups. The patients who were diagnosed with COPD upon admission to the ICU were predisposed to infection with IRAB $(\mathrm{P}=0.028)$. The hospital mortality rate in the case group was higher than in the control group $(\mathrm{P}=0.032)$ (Table 1$)$.

\section{Environmental and hand surveillance}

In August 23, 310 environmental samples were collected. Nine (2.90\%) samples were found to be culture positive for IRAB. These strains were isolated from computer keyboards $(n=3)$, bed rails $(n=3)$, nurses' supply carts $(n=2)$, and a ventilator control panel $(n=1)$. The distribution of culture samples positive for IRAB from the environment and from patients is shown in Figure 3.

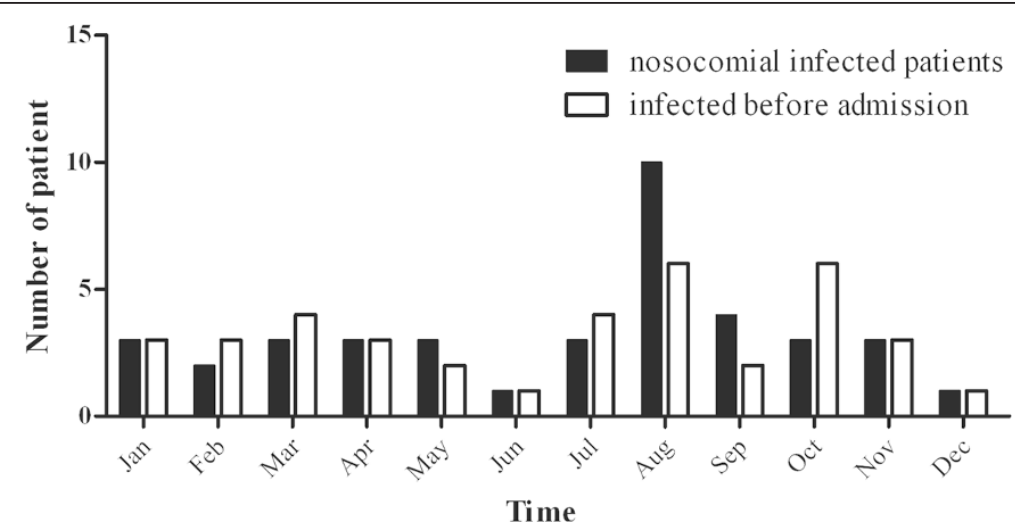

Figure 3 Monthly report of new cases of IRAB infection. 
Table 1 Demographic and clinical characteristics of case patients infected with IRAB, compared with non-infected control patients

\begin{tabular}{|c|c|c|c|}
\hline Characteristic $^{a}$ & $\begin{array}{l}\text { Cases } \\
(n=11)\end{array}$ & $\begin{array}{l}\text { Controls } \\
(n=14)\end{array}$ & $P$ value \\
\hline Age (years) & $65.09 \pm 12.65$ & $63.86 \pm 14.33$ & 0.824 \\
\hline Gender, male (\%) & $5(45.46)$ & $5(35.71)$ & 0.622 \\
\hline Prior hospital stay ( $<1$ year) & $3(27.27)$ & $2(14.29)$ & 0.420 \\
\hline Length of stay in ICU (days) & $6(3-25)$ & $11(2.75-25.75)$ & 0.784 \\
\hline APACHE II score upon ICU admission & $21.82 \pm 7.13$ & $21.50 \pm 8.04$ & 0.919 \\
\hline SOFA score upon ICU admission & $6.55 \pm 3.14$ & $7.00 \pm 6.49$ & 0.762 \\
\hline \multicolumn{4}{|l|}{ Primary ICU admission diagnosis } \\
\hline Chronic pulmonary disease & $8(72.72)$ & $4(28.57)$ & 0.028 \\
\hline Pneumonia & $8(72.72)$ & $8(57.14)$ & 0.42 \\
\hline Diabetes mellitus & $1(9.09)$ & $2(14.28)$ & 0.692 \\
\hline Hypertension & $2(18.18)$ & $5(35.71)$ & 0.332 \\
\hline Malignancy & $2(18.18)$ & $3(21.42)$ & 0.84 \\
\hline Surgery procedure (<30 days) & $1(9.09)$ & $5(35.71)$ & 0.122 \\
\hline \multicolumn{4}{|l|}{ Antibiotic classes $^{\text {b }}$} \\
\hline Carbapenem & $7(63.63)$ & $6(42.86)$ & 0.302 \\
\hline Penicillins & $3(27.27)$ & $6(42.56)$ & 0.420 \\
\hline Piperacillin/tazobactam & $5(45.45)$ & $6(42.86)$ & 0.897 \\
\hline Cefepime & $7(63.63)$ & $6(42.86)$ & 0.302 \\
\hline Fluoroquinolones & $3(27.28)$ & $3(21.42)$ & 0.734 \\
\hline Glycopeptide & $6(54.54)$ & $8(57.14)$ & 0.716 \\
\hline Antifungal & $4(36.36)$ & $4(28.57)$ & 0.678 \\
\hline \multicolumn{4}{|l|}{ ICU procedures } \\
\hline Ventilator & $11(100)$ & $14(100)$ & - \\
\hline Blood transfusion & $5(45.45)$ & $7(50)$ & 0.821 \\
\hline Hemodialysis & $0(0)$ & $4(28.57)$ & 0.053 \\
\hline Nasogastric tube & $11(100)$ & $12(85.71)$ & 0.191 \\
\hline Venous central catheter & $10(81.82)$ & $13(92.86)$ & 0.859 \\
\hline Peripheral venous catheter & $11(100)$ & $13(92.86)$ & 0.366 \\
\hline Arterial catheter & $4(36.36)$ & $6(42.86)$ & 0.742 \\
\hline Urinary catheter & $11(100)$ & $14(100)$ & - \\
\hline \multicolumn{4}{|l|}{ Outcome } \\
\hline 28-day mortality & $3(27.27)$ & $2(14.29)$ & 0.420 \\
\hline Hospital mortality & $6(54.56)$ & $3(21.42)$ & 0.032 \\
\hline
\end{tabular}

${ }^{\mathrm{a}}$ All data are presented as the number, with the percentage in parenthesis, with the exception of age, APACHE II score, and SOFA score, which are presented as mean \pm standard deviation. Length of ICU stay was presented as median (interquartile range).

${ }^{\mathrm{b}}$ Case group: used within 30 days before the first IRAB isolate was discovered and the antibiotics had been used for at least $72 \mathrm{~h}$. Control group: antibiotics had been used in the ICU for at least $72 \mathrm{~h}$.

Among 15 samples from the hands (or gloves) of 15 hospital care workers (doctors, nurses, and cleaners), four (26.67\%) tested positive for IRAB; one of these was from the hands of a hospital care worker and three were from gloves. The samples from the HCW's hands were collected without forewarning, and some were collected immediately after the HCW had been in direct contact with patients.
As a result of the continuing number of cases being reported, an environmental survey was conducted in November and December 2011. In November, 620 environmental samples were collected. At the same time, $10(1.61 \%)$ samples were collected from the hands (or gloves) of hospital care workers. Twenty-one isolates were found to be culture positive for IRAB. These 
strains were isolated from bed rails $(n=3)$, computer keyboards $(n=2)$, curtains $(n=1)$, ventilator air flow sensor $(n=1)$, ventilator control panel $(n=1)$, supply cart $(n=1)$, and a bedside cabinet $(n=1)$. No samples from the hands (or gloves) tested positive for IRAB. In December, 620 environmental samples were collected, as well as samples from the hands (or gloves) of 10 hospital care workers, but only one isolate from a mattress was found to be culture positive for IRAB.

\section{Molecular typing of IRAB clinical and environmental isolates}

We analyzed molecular typing of 39 strains, including 24 isolated from the environment in August, November, and December 2011; 15 of which were isolated from clinical samples during the environmental survey. DiversiLab revealed seven clones (designated A-G) (Figure 4). The most common was Clone $\mathrm{D}$, which accounted for $16(41.02 \%)$ of the 39 isolates. Clone D included eight clinical isolates, seven environmental strains, and one isolate from an HCW's hand. These data suggest that Clone D was the predominant clone of the outbreak in the ICU.

\section{Discussion}

Since 2008, there have been reports of ICU outbreaks caused by IRAB in our ICU [14]. The isolation rate of $A$. baumannii was $39.90 \%$ and the imipenem-resistance rate was $85 \%$; ISAbal-associated bla $a_{\text {OXA-23 }}$ genes were prevalent in IRAB, with an isolation rate of $82.5 \%$ in 2011 (our unpublished data). Several IRAB nosocomial outbreaks with the ISAba1-associated bla $a_{\mathrm{OXA}-23}$ resistance mechanism have been reported [15]. Prevention of epidemic outbreaks of $A$. baumannii is a challenging task in our ICU.

It has been reported that computer keyboards and curtains are contaminated with bacterial pathogens, which can act as reservoirs of nosocomial pathogens [16-18]. Bed rails and supply carts are considered to be high-touch surfaces [19]. Furthermore, bed rails have been previously shown to have the highest frequency of contamination with $A$. baumannii [20,21]. In the present outbreak, environmental survey results revealed that bed rails were the objects most frequently found to be contaminated, followed by computer keyboards, and supply carts. Our findings were consistent with previously published data.

Covert observation found that some doctors and nurses wear gloves when contacting clean area items, such as operating the ventilator and computers. Therefore, we speculated that gloved HCWs contaminated equipment (such as computer keyboards, supply carts, and ventilator panels) with IRAB and disseminated the outbreak strains in the ICU. Swab cultures from the hands (or gloves) of HCWs found IRAB, and DiversiLab analysis showed that the strains isolated from the hands (or gloves), equipment, and clinical samples were Clones D-F. We identified transient hand (or glove) carriage, and confirmed that cross-transmission of IRAB was involved in hand contamination. We suggest that equipment was contaminated via the hands or contaminated gloves of HCWs. These data further suggest that the environment may be secondarily contaminated by patients, who then become a potential reservoir [22].

We impose financial penalties to prohibit contact the cleaning equipment with gloves. From the environmental culture results in December 2011, this intervention was efficient at preventing IRAB contamination, and the detection rate in environmental culture decreased from $2.90 \%(9 / 310)$ to $0.16 \%(1 / 620)$. This observation demonstrated that HCWs tend to wear gloves as a means of self-protection, rather than to prevent cross-infection of patients, which is consistent with hand hygiene using an alcohol-based hand rub [23].

We also analyzed homology of IRAB recovered from patients during the environmental survey. On August 23,2011 , nine infected patients stayed in the ICU. DiversiLab data demonstrated that six strains (P3, 9, 8, 10, 12, and 13 August) recovered from these patients belonged to Clone D. Five of these strains were isolated from nosocomially infected patients. One strain (P3-Aug) was recovered from a patient admitted on July 22, 2011, who had been infected with IRAB before admission, and cultured positively throughout admission. The remaining three clinical isolates (P17, 18, and 19 August) belonged to different clones that were isolated from patients infected with IRAB before admission and were transferred from another hospital. Therefore, we speculated that the original outbreak may have been from the patient with P3-Aug.

Epidemiological typing results suggested that the ICU had four epidemic clones (D-G) in the outbreak. The August clones remained in the environment and infected patients through to December 2011. It may be difficult to eradicate IRAB in ICU outbreaks [22], especially without closing ICU even with improved infection control practice $[24,25]$. Our intervention decreased the number of contaminated surfaces and resulted in a sustained reduction in the number of IRAB-infected patients.

Reduction the rate of nosocomial infection by surveillance and prevention program could be benefited from the Hawthorn effect [26,27]. Successful control outbreak depends on the cooperation of the ward staff [27]. In the present outbreak, covert observation had been executed which would influence the behavior of ICU staff. The outbreak control team including ICU doctors and nurse except bacteriologist and infection control officer. This can create a sense of ownership of infection control and then form cooperation among them. 


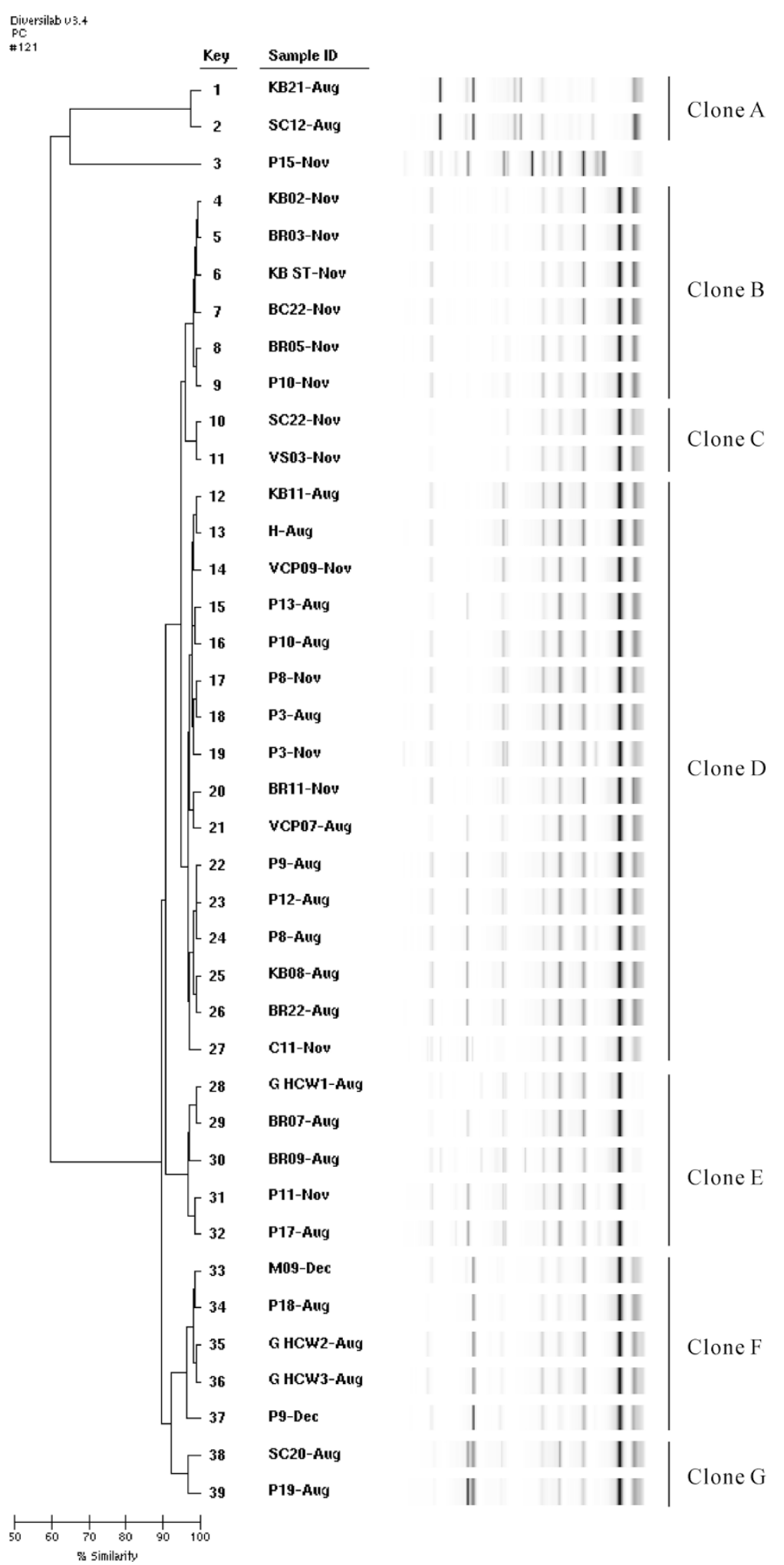

Figure 4 Rep-PCR-based similarity analysis and gel images for 39 IRAB isolates from clinical and environmental samples. Number presents the nember of beds. BC: bedside cabinet; BR: bed rail; C: curtain; G: gloves; H: hand; KB: computer keyboard; KB ST: computer keyboard at nurses' station; M: mattress; P: patient; SC: supply cart; VCP: ventilator control panel; VS: ventilator air flow sensor. 
Complete closure of clinical units has been recommended for outbreak control in some previous studies $[28,29]$, but not in others $[12,30,31]$. In our setting, there was no available ICU to transfer the infected patients and we needed to keep the unit open. Therefore, our patients were immediately transferred out from the ICU once they were clinically stabilized, to reduce infection with IRAB and HCW workload.

Patients admitted to our ICU are often already infected (severe pneumonia was present in 16/25 patients). To control the outbreak, we implemented aggressive interventions of contact isolation for all patients after their admission, and the contact isolation was maintained until a negative culture result was obtained. By using enforced contact isolation, the chance of pathogens spreading during the time from patients' admission to contact isolation can be reduced.

The present studies had some limitations. We demonstrated that the environment may be secondarily contaminated by the patients. To find the source of infection may need a long period of monitoring. The effects of HCW workload and the cost of extra cleaning were not investigated.

\section{Conclusion}

The outbreak was controlled by a multidisciplinary approach. The study highlights the importance of glove removal. Contact isolation for all patients at admission may be an effective measure to avoid outbreaks of $A$. baumannii in endemic areas.

\section{Abbreviations}

ICU: Intensive care units; IRAB: Imipenem-resistant Acinetobacter baumannii; rep-PCR: repetitive extragenic palindromic sequence-based PCR;

HCWs: Healthcare workers; COPD: Chronic obstructive pulmonary disease.

\section{Competing interests}

The authors declare that they have no competing interests.

\section{Authors' contributions}

DY and JS carried out the isolation and molecular typing. JL and PM analyzed and interpreted data and made contributions to draft of this manuscript. YH performed the statistical analysis. DY and $\mathrm{CL}$ participated in the environment survey and infection control interventions. XL, WH participated in the collection demographic and clinical data and participated in the infection control interventions. YL and PM contributed to the design, coordination and revision the manuscript. All authors read and approved the final manuscript.

\section{Acknowledgments}

The authors would like to express their appreciation to Professor Cheng Chi Chung (The University of Hong Kong, Queen Mary Hospital) for suggestions and editorial assistance. This work was funded by grants from the Science and Technology Project of Guangzhou (2010 J-E171).

\section{Author details}

'Department of Infection Control, The First Affiliated Hospital of Guangzhou medical university, Guangzhou, Guangdong 510120, China. ${ }^{2}$ State Key Laboratory of Respiratory Disease, The First Affiliated Hospital of Guangzhou medical university, Guangzhou, Guangdong 510120, China. Intensive Care Unit, The First Affiliated Hospital of Guangzhou medical university, Guangzhou, Guangdong 510120, China.
Received: 12 January 2015 Accepted: 31 March 2015

Published online: 11 April 2015

\section{References}

1. Durante-Mangoni E, Zarrilli R. Global spread of drug-resistant Acinetobacter baumannii: molecular epidemiology and management of antimicrobial resistance. Future Microbiol. 2011;6(4):407-22.

2. Bergogne-Berezin E, Towner KJ. Acinetobacter spp. as nosocomial pathogens: microbiological, clinical, and epidemiological features. Clin Microbiol Rev. 1996;9(2):148-65.

3. Boyce JM. Environmental contamination makes an important contribution to hospital infection. J Hosp Infect. 2007;65 Suppl 2:50-4.

4. Weber DJ, Rutala WA, Miller MB, Huslage K, Sickbert-Bennett E. Role of hospital surfaces in the transmission of emerging health care-associated pathogens: norovirus, Clostridium difficile, and Acinetobacter species. Am J Infect Control. 2010;38(5 Suppl 1):S25-33.

5. Dijkshoorn L, Nemec A, Seifert H. An increasing threat in hospitals: multidrug-resistant Acinetobacter baumannii. Nat Rev Microbiol. 2007;5(12):939-51.

6. Perez F, Hujer AM, Hujer KM, Decker BK, Rather PN, Bonomo RA. Global challenge of multidrug-resistant Acinetobacter baumannii. Antimicrob Agents Chemother. 2007;51(10):3471-84

7. Sievert DM, Ricks P, Edwards JR, Schneider A, Patel J, Srinivasan A, et al. Antimicrobial-resistant pathogens associated with healthcare-associated infections: summary of data reported to the National Healthcare Safety Network at the Centers for Disease Control and Prevention, 2009-2010. Infect Control Hosp Epidemiol. 2013;34(1):1-14.

8. Lee YT, Fung CP, Wang FD, Chen CP, Chen TL, Cho WL. Outbreak of imipenem-resistant Acinetobacter calcoaceticus-Acinetobacter baumannii complex harboring different carbapenemase gene-associated genetic structures in an intensive care unit. J Microbiol Immunol Infect. 2012;45(1):43-51.

9. Garlantezec R, Bourigault C, Boles JM, Prat G, Baron R, Tonnelier JM, et al. Investigation and management of an imipenem-resistant oxa-23 Acinetobacter baumannii outbreak in an intensive care unit. Med Mal Infect. 2011:41(8):430-6.

10. Consales G, Gramigni E, Zamidei L, Bettocchi D, De Gaudio AR. A multidrug-resistant Acinetobacter baumannii outbreak in intensive care unit: antimicrobial and organizational strategies. J Crit Care. 2011;26(5):453-9.

11. Chmielarczyk A, Higgins PG, Wojkowska-Mach J, Synowiec E, Zander E, Romaniszyn D, et al. Control of an outbreak of Acinetobacter baumannii infections using vaporized hydrogen peroxide. J Hosp Infect. 2012;81 (4):239-45.

12. Orsi GB, Franchi C, Giordano A, Rocco M, Ferraro F, Mancini C, et al. Multidrug-resistant Acinetobacter baumannii outbreak in an intensive care unit. J Chemother. 2008;20(2):219-24.

13. Garner JS, Jarvis WR, Emori TG, Horan TC, Hughes JM. CDC definitions for nosocomial infections. In: Olmsted RN, editor. APIC infection Control and Applied Epidemiology: Principles and Practice. St. Louis: Mosby; 1996. p. A1-A20.

14. Pu M, Dan $\mathrm{Y}$, Song-lin $\mathrm{W}$, Hong-chuan $\mathrm{H}$, Xiao-qing $\mathrm{L}$, Wei-qun $\mathrm{H}$, et al. homology of sequential prevalence of multidrug-resistant acinetobacter baumannii in intensive care unit. chinese journal of nosocomiology. 2011;21(9):1741-6.

15. Pu M, Gui-xia Q, Dan Y, Xiao-qing L, Yi-min L. The resistence mechanism and molecular epidemiology of carbapenem-resistant acinetobacter baumannii in an intensive care unit. Chin J Infect Chemother. 2012;12(6):449-52.

16. Bures S, Fishbain JT, Uyehara CF, Parker JM, Berg BW. Computer keyboards and faucet handles as reservoirs of nosocomial pathogens in the intensive care unit. Am J Infect Control. 2000;28(6):465-71.

17. Catano JC, Echeverri LM, Szela C. Bacterial contamination of clothes and environmental items in a third-level hospital in Colombia. Interdisciplinary perspectives on infectious diseases. 2012;2012:507640.

18. Otter JA, Yezli S, French GL. The role played by contaminated surfaces in the transmission of nosocomial pathogens. Infect Control Hosp Epidemiol. 2011;32(7):687-99.

19. Huslage K, Rutala WA, Sickbert-Bennett E, Weber DJ. A quantitative approach to defining "high-touch" surfaces in hospitals. Infect Control Hosp Epidemiol. 2010;31(8):850-3.

20. Neely AN, Maley MP, Warden GD. Computer keyboards as reservoirs for Acinetobacter baumannii in a burn hospital. Clin Infect Dis. 1999;29(5):1358-60.

21. Thom KA, Johnson JK, Lee MS, Harris AD. Environmental contamination because of multidrug-resistant Acinetobacter baumannii surrounding colonized or infected patients. Am J Infect Control. 2011;39(9):711-5. 
22. Chang HL, Tang CH, Hsu YM, Wan L, Chang YF, Lin CT, et al. Nosocomial outbreak of infection with multidrug-resistant Acinetobacter baumannii in a medical center in Taiwan. Infect Control Hosp Epidemiol. 2009;30(1):34-8.

23. Monistrol O, Calbo E, Riera M, Nicolas C, Font R, Freixas N, et al. Impact of a hand hygiene educational programme on hospital-acquired infections in medical wards. Clin Microbiol Infect. 2012;18(12):1212-8.

24. Munoz-Price LS, Namias N, Cleary T, Fajardo-Aquino Y, Depascale D, Arheart $\mathrm{KL}$, et al. Acinetobacter baumannii: association between environmental contamination of patient rooms and occupant status. Infect Control Hosp Epidemiol. 2013;34(5):517-20.

25. Guerrero DM, Carling PC, Jury LA, Ponnada S, Nerandzic MM, Donskey CJ. Beyond the Hawthorne effect: reduction of Clostridium difficile environmental contamination through active intervention to improve cleaning practices. Infect Control Hosp Epidemiol. 2013;34(5):524-6.

26. Burke JP. Surveillance, reporting, automation, and interventional epidemiology. Infect Control Hosp Epidemiol. 2003;24(1):10-2.

27. Orsi GB, Raponi M, Franchi C, Rocco M, Mancini C, Venditti M. Surveillance and infection control in an intensive care unit. Infect Control Hosp Epidemiol. 2005;26(3):321-5

28. Markogiannakis A, Fildisis G, Tsiplakou S, Ikonomidis A, Koutsoukou A, Pournaras $\mathrm{S}$, et al. Cross-transmission of multidrug-resistant Acinetobacter baumannii clonal strains causing episodes of sepsis in a trauma intensive care unit. Infect Control Hosp Epidemiol. 2008;29(5):410-7.

29. Maragakis LL, Cosgrove SE, Song X, Kim D, Rosenbaum P, Ciesla N, et al. An outbreak of multidrug-resistant Acinetobacter baumannii associated with pulsatile lavage wound treatment. JAMA. 2004;292(24):3006-11.

30. Wilks M, Wilson A, Warwick S, Price E, Kennedy D, Ely A, et al. Control of an outbreak of multidrug-resistant Acinetobacter baumannii-calcoaceticus colonization and infection in an intensive care unit (ICU) without closing the ICU or placing patients in isolation. Infect Control Hosp Epidemiol. 2006;27(7):654-8

31. Hosoglu S, Hascuhadar M, Yasar E, Uslu S, Aldudak B. Control of an Acinetobacter baumannii outbreak in a neonatal ICU without suspension of service: a devastating outbreak in Diyarbakir, Turkey. Infection. 2012:40(1):11-8.

\section{Submit your next manuscript to BioMed Central and take full advantage of:}

- Convenient online submission

- Thorough peer review

- No space constraints or color figure charges

- Immediate publication on acceptance

- Inclusion in PubMed, CAS, Scopus and Google Scholar

- Research which is freely available for redistribution 